\title{
Prediction Interval Development for Wind-Tunnel
}

\section{Balance Check-Loading}

\author{
Drew Landman ${ }^{1}$ and Kenneth G. Toro ${ }^{2}$ \\ Old Dominion University, Norfolk, VA 23529 \\ Sean A. Commo ${ }^{3}$ and Keith C. Lynn ${ }^{4}$ \\ NASA Langley Research Center, Hampton, Virginia 23681
}

Results from the Facility Analysis Verification and Operational Reliability project revealed a critical gap in capability in ground-based aeronautics research applications. Without a standardized process for check-loading the wind-tunnel balance or the model system, the quality of the aerodynamic force data collected varied significantly between facilities. A prediction interval is required in order to confirm a check-loading. The prediction interval provides an expected upper and lower bound on balance load prediction at a given confidence level. A method has been developed which accounts for sources of variability due to calibration and check-load application. The prediction interval method of calculation and a case study demonstrating its use is provided. Validation of the methods is demonstrated for the case study based on the probability of capture of confirmation points.

${ }^{1}$ Professor, Department of Mechanical and Aerospace Engineering, 1300 Elkhorn Avenue, Norfolk, VA 23529, AIAA Associate Fellow.

2 Graduate Research Assistant, Department of Mechanical and Aerospace Engineering, 1300 Elkhorn Avenue, Norfolk, VA 23529, AIAA Student Member.

3 Research Engineer, Systems Engineering and Engineering Methods Branch, MS 131, Hampton, VA 23681 and AIAA member.

${ }^{4}$ Research Engineer, Aeronautics Systems Engineering Branch, MS 238, Hampton, VA 23681 and AIAA member. 


\section{Introduction}

A wind-tunnel test program often involves a complicated model installation process which may include installation of hardware that bridges the metric to non-metric gap. Clearances between non-metric supports and the balance supported test article may involve tight tolerances such that support deflections under aerodynamic loading or large environmental changes that may lead to contact. Even in the case of a simple installation, it is always prudent to apply known forces and moments to a model/balance and observe that these loads are being correctly measured prior to testing - a check-loading [1]. Current practices for confirmation of check-loads are typically informal and involve using the standard deviation of the calibration residuals with a coverage factor based on the desired confidence. A more mathematically defensible bound on the prediction of loads may be provided by a prediction interval, which contains two general sources of uncertainty. The first is that due to the balance uncertainty obtained during the balance calibration. The second is the uncertainty in the applied loads created by the check-load hardware.

\section{Estimates of Uncertainty in Balance Responses}

Estimates of uncertainty in load measurement from the balance are obtained during the calibration process. Estimates include the uncertainty in setting the applied loads due to the calibration hardware used, as well as the error associated with the balance reading of the loads through the calibration model. Set-point errors as well as any measurement system error are also included in these estimates. A rigorous, statistical approach to the calibration process provides an estimate for the variance in each of the component bridge voltage responses through the Mean Square for Error (MSE) available from the Analysis of Variance (ANOVA). The developed regression model depends on the design point load combinations, expressed here in coded units $(x)$. A typical model used with monolithic moment balances contains at least primary sensitivities, 1st order interactions, two-factor interactions, and pure quadratics as shown in Eq. (1) [1].

$$
y_{k}=\beta_{0}+\sum_{i=1}^{6} \beta_{i} x_{i}+\sum_{j=1}^{6} \sum_{i<j} \beta_{i j} x_{i} x_{j}+\sum_{i=1}^{6} \beta_{i i} x_{i}^{2}+\epsilon
$$

The response is the bridge voltage of the component of interest, $y$. The $x$ 's in the model represent the applied loads given in coded factors. Each estimated term in the regression model $\left(\beta_{i}\right)$ is 
represented as a column in the calibration model matrix $\mathbf{X}$ used during the regression calculations. The calibration model matrix $\mathbf{X}$ contains only statistically significant terms, tested at the desired significance level $(\alpha)$. One challenge faced in using calibration models is that the user ultimately requires the output in force and moment engineering units, not voltages. The calibration model is $y=f(x)$ whereas the desired output is $x=f(y)$.

A prediction interval is a bound on the predicted response for a given applied loading - for instance a confirmation point during calibration. The vector $\mathbf{x}_{0}$ is used to define a single multicomponent load combination, expanded to include all terms in the calibration regression model. The t-statistic is computed for a given level of confidence $(1-\alpha)$, and $(n-p)$ residual degrees of freedom. There are $n$ unique calibration design points (or load combinations) in the calibration design and $p$ parameters in the resulting regression model. The prediction interval for a single confirmation point evaluated during calibration may then be expressed as Eq. (2) [2, 3].

$$
\hat{y}\left(\mathbf{x}_{0}\right) \pm t_{\alpha / 2, n-p} \sqrt{\operatorname{MSE}\left(1+\mathbf{x}_{0}^{\prime}\left(\mathbf{X}^{\prime} \mathbf{X}\right)^{-1} \mathbf{x}_{0}\right)}
$$

This prediction interval for response voltage can then be expressed as an upper and lower bound for forces and moments by computing the loads through the balance calibration model. This model is self-consistent for use during calibration, but the bias error (expressed as a variance) associated with the uncertainty in applied loads due to the calibration hardware $\left(\sigma_{\text {bias-cal }}^{2}\right)$ must be added to the random component (MSE) for the overall uncertainty $[4,5]$. Note that the bias must be expressed as a voltage here for unit consistency.

$$
\hat{y}\left(\mathbf{x}_{0}\right) \pm t_{\alpha / 2, n-p} \sqrt{\left(\mathrm{MSE}+\sigma_{\text {bias-cal }}^{2}\right)\left(1+\mathbf{x}_{0}^{\prime}\left(\mathbf{X}^{\prime} \mathbf{X}\right)^{-1} \mathbf{x}_{0}\right)}
$$

\section{Uncertainty in Loads Applied by the Check-Load Hardware}

The check-loading hardware is used to apply known total loads to the balance/model including the loads due to the hardware. The mass properties of the check-load hardware and all dimensions that define the moment arms should be measured with the highest possible precision. A software program can then be developed for incorporating these precise measurements to calculate the loads 
applied to the test article by the check-load hardware (including tare weights). A method is now required to combine all the individual uncertainties. A Monte Carlo simulation allows integration of all individual uncertainties including all dimension tolerances, and the uncertainty in the applied and tare weights [4]. An estimate of the standard deviation is required for each source of uncertainty. The Monte Carlo simulation models each error source as a normal distribution using the mean value of the parameter and standard deviations. Overall standard deviations in each response are then calculated for each of the runs to provide an absolute bias estimate expressed in units of response voltage for the applied loads $\left(\sigma_{\text {bias-applied }}^{2}\right)$. Details are shown in the case study that follows.

\section{Check-Load Prediction Interval for Total Uncertainty}

The prediction interval used for check-loading expressed as a balance bridge output is given as Eq. (4). It is based on the development presented in Reference 3 but includes the additional bias due to the applied loads via the check-load hardware and the bias from the calibration [5].

$$
\hat{y}\left(\mathbf{x}_{0}\right) \pm t_{\alpha / 2, n-p} \sqrt{\left(\sigma_{\text {bias-applied }}^{2}+\mathrm{MSE}+\sigma_{\text {bias-cal }}^{2}\right)\left(1+\mathbf{x}_{0}^{\prime}\left(\mathbf{X}^{\prime} \mathbf{X}\right)^{-1} \mathbf{x}_{0}\right)}
$$

This interval now allows the user to choose a load combination, apply it through the check-load hardware, read the balance response and determine if this response captures the applied load in the prediction interval. The balance response is a voltage and many may feel that it is easier to interpret a response in the engineering units for a force and moment. The calibration matrix may be used to back calculate the force and moment values in engineering units using the method outlined in Reference 1.

\section{Case Study: The NASA Langley In-Situ Load System}

Specialized hardware has recently been developed at NASA Langley Research Center (LaRC) to provide rapid, in-situ validation of wind-tunnel model balance loads in the wind tunnel just prior to testing. The case study presented here coupled this new hardware with a typical balance used in the NASA LaRC National Transonic Facility (NTF). The study involved loading the balance using a calibration fixture on the metric end while supporting the balance on a system capable of adjusting both pitch and roll orientation on the non-metric end. 


\section{A. The ILS Concept}

The In-Situ Load System (ILS) is a new system designed at NASA LaRC to help address the issues surrounding a system-level validation or calibration of a wind-tunnel model system (WTMS) [6]. Together, the aircraft model, model sting, balance, angle measurement system, and other instrumentation make up the WTMS. The ILS incorporates the rigorous methodology described above for quantifying uncertainties to standardize the check-loading process. The ILS concept has origins in the NASA LaRC Single-Vector Calibration System (SVS), which exploits the use of a single deadweight loading to create variable, multi-component loads through rotation and offset of the point of load application with respect to the balance moment center (BMC) [7]. Fig. 1 shows the ILS and illustrates the principles of operation. The ILS has a two degree-of-freedom joint which

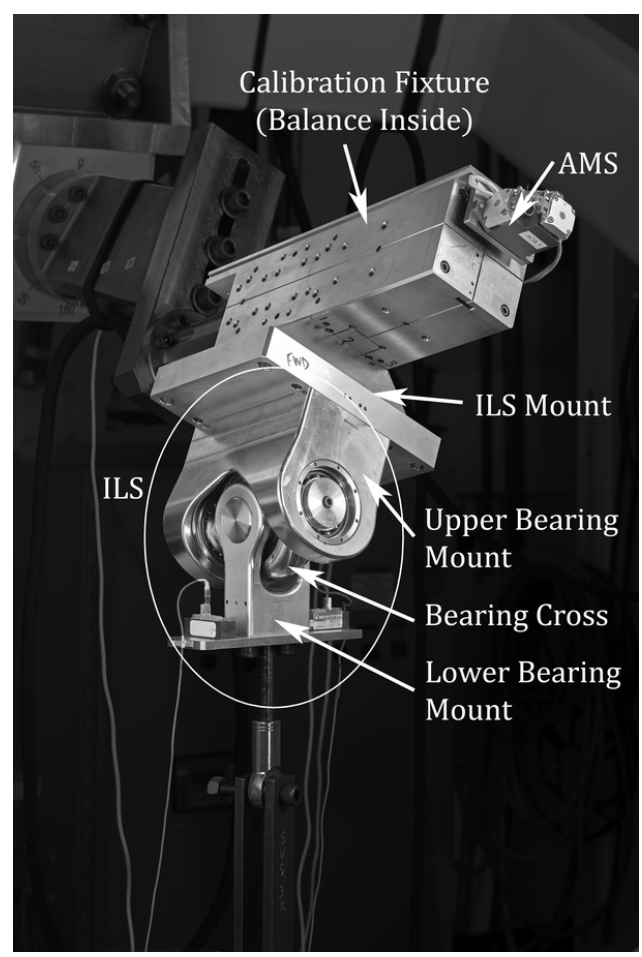

Fig. 1 The ILS Mounted to a Balance Calibration Fixture

allows the applied load to remain aligned with the gravitational vector. The upper bearing mount supports two bearings which in turn support the bearing cross shaft. The bearing cross contains a third bearing which is tied to the lower bearing mount. Loads are applied through a weight hanger attached to the lower bearing mount. For the purpose of this study, the ILS was attached directly 
to a balance calibration fixture. Offsets in the $x$ - and $y$-direction are afforded through multiple mounting holes in the ILS mount and the fixture (axes shown in Fig. 2). The balance is inserted in the fixture block and supported and oriented on the non-metric end by allowing movement in both the pitch and roll axes. An Angle Measurement System (AMS) is installed to the front of the calibration fixture to provide the orientation of the balance with respect to the gravitational vector. The AMS package consists of three Q-flex accelerometers that are oriented orthogonally [8].

\section{B. Loads Applied by the ILS}

The design of the ILS hardware ensures that the load vector coincides with the gravity vector. Based on the balance coordinate system, the load vector is resolved into the three forces. $\mathbf{F}_{b a l}$ is the vector of the three forces defined as $\left[\begin{array}{lll}F_{x} & F_{y} & F_{z}\end{array}\right]^{\prime}$.

$$
\mathbf{F}_{\text {bal }}=F_{a p p} \mathbf{g}
$$

where $F_{a p p}$ is the magnitude of the applied load vector, and $\mathbf{g}$ is the gravity vector describing the orientation of the balance expressed as $\left[g_{x} g_{y} g_{z}\right]^{\prime}$. For a constant $F_{a p p}$, the magnitude of the three forces is varied by changing the orientation of the balance in pitch and roll. The components of the gravity vector are expressed in terms of $g$ 's. The moments applied to the balance are not only a

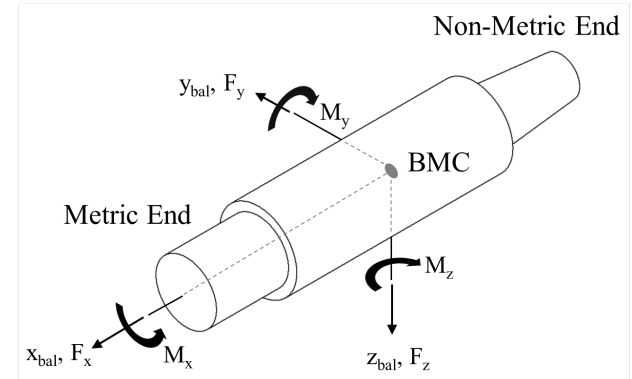

Fig. 2 Balance Moment Center Reference Axes

function of the applied load vector and the orientation of the balance, but also the distance of the load point from the BMC. The BMC is an imaginary point on the balance that is used to define the balance coordinate frame, and is the point by which all forces and moments are referenced. The distance from the BMC is a vector expressed as $\mathbf{d}_{\mathrm{BMC}}=\left[x_{B M C} y_{B M C} z_{B M C}\right]^{\prime}$, where the sign 
convention is defined in Fig. 2. The applied moments about BMC, $\mathbf{M}_{b a l}$, are the cross product of the distance vector and the balance force vector, or

$$
\mathbf{M}_{\mathrm{bal}}=\mathrm{d}_{\mathrm{BMC}} \times \mathbf{F}_{\mathrm{bal}}
$$

where $\mathbf{M}_{\mathbf{b a l}}$ is the vector of the three moments defined as $\left[M_{x} M_{y} M_{z}\right]^{\prime}$ and $\mathbf{F}_{\text {bal }}$ is given by Eq. 5 .

\section{The NTF-113C Balance}

The NTF-113C balance is a single-piece, six-component moment balance designed for full-span testing at the NTF. Table 1 shows the full-scale design loads for the NTF-113C. The NTF-113C

Table 1 NTF-113C Balance Design Loads

\begin{tabular}{cccc}
\hline \hline Balance & Aerodynamic & \multicolumn{2}{c}{ Design Load } \\
Component & Component & English Units, lbs. or in-lbs. & Metric Units, N or N-m \\
\hline$F_{z}$ & $N F$ & 6,520 & 29,002 \\
$F_{x}$ & $A F$ & 400 & 1,779 \\
$M_{y}$ & $P M$ & 12,800 & 1,446 \\
$M_{x}$ & $R M$ & 8,150 & 920 \\
$M_{z}$ & $Y M$ & 6,400 & 723 \\
$F_{y}$ & $S F$ & 4,000 & 17,792 \\
\hline \hline
\end{tabular}

was selected for this study since the ILS has a maximum applied load limit of 5,000 lbs., which is 77 percent of the full-scale capacity of normal force. Full-scale loads are possible with the remaining five components using the ILS. The bridge electrical configuration is such that the strain-gage bridges are in a force-balance format (NF1/NF2, SF1/SF2, RM, AF), where the beam elements are located in two cages equally spaced at axial stations forward and aft of the BMC as shown in the overview drawing of Fig. 3

\section{The ILS Check-Load Study}

The load schedule shown in Table 2 was used in testing the ILS hardware with the NTF-113C balance. The applied load was fixed at 2,500 lbs for the testing since a lower value of $F_{a p p}$ allowed 


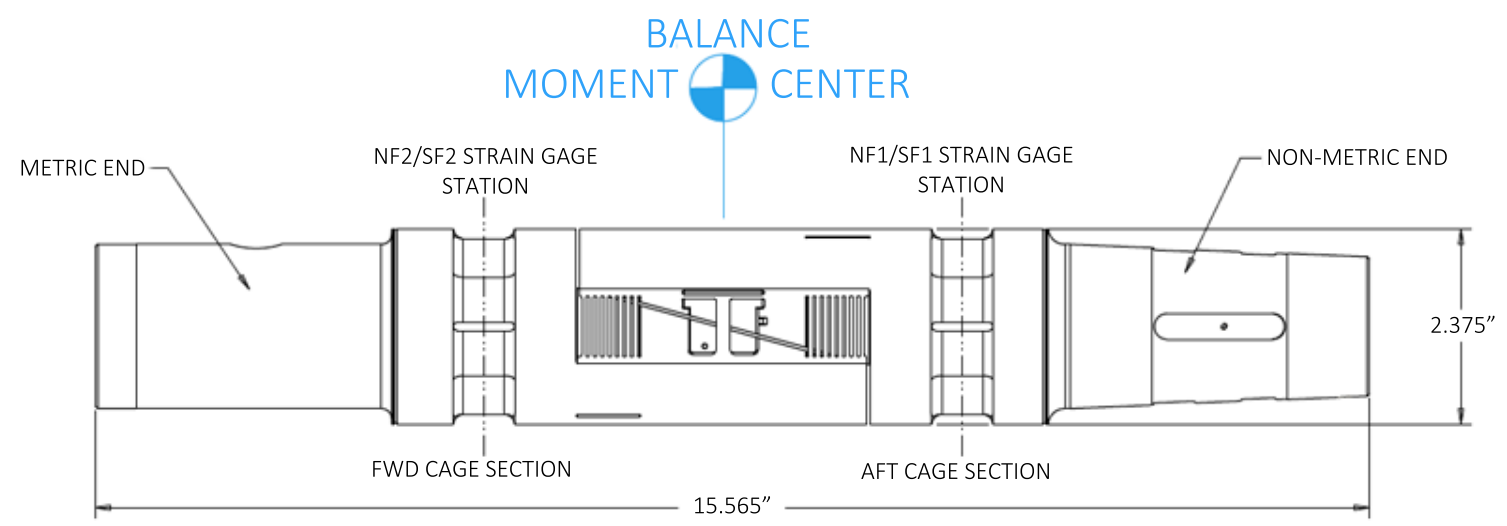

Fig. 3 NTF-113C Balance Overview

for a larger volume of the six-dimensional space to be explored. By increasing $F_{a p p}$, the design

Table 2 ILS Check-Load Schedule for NTF-113C

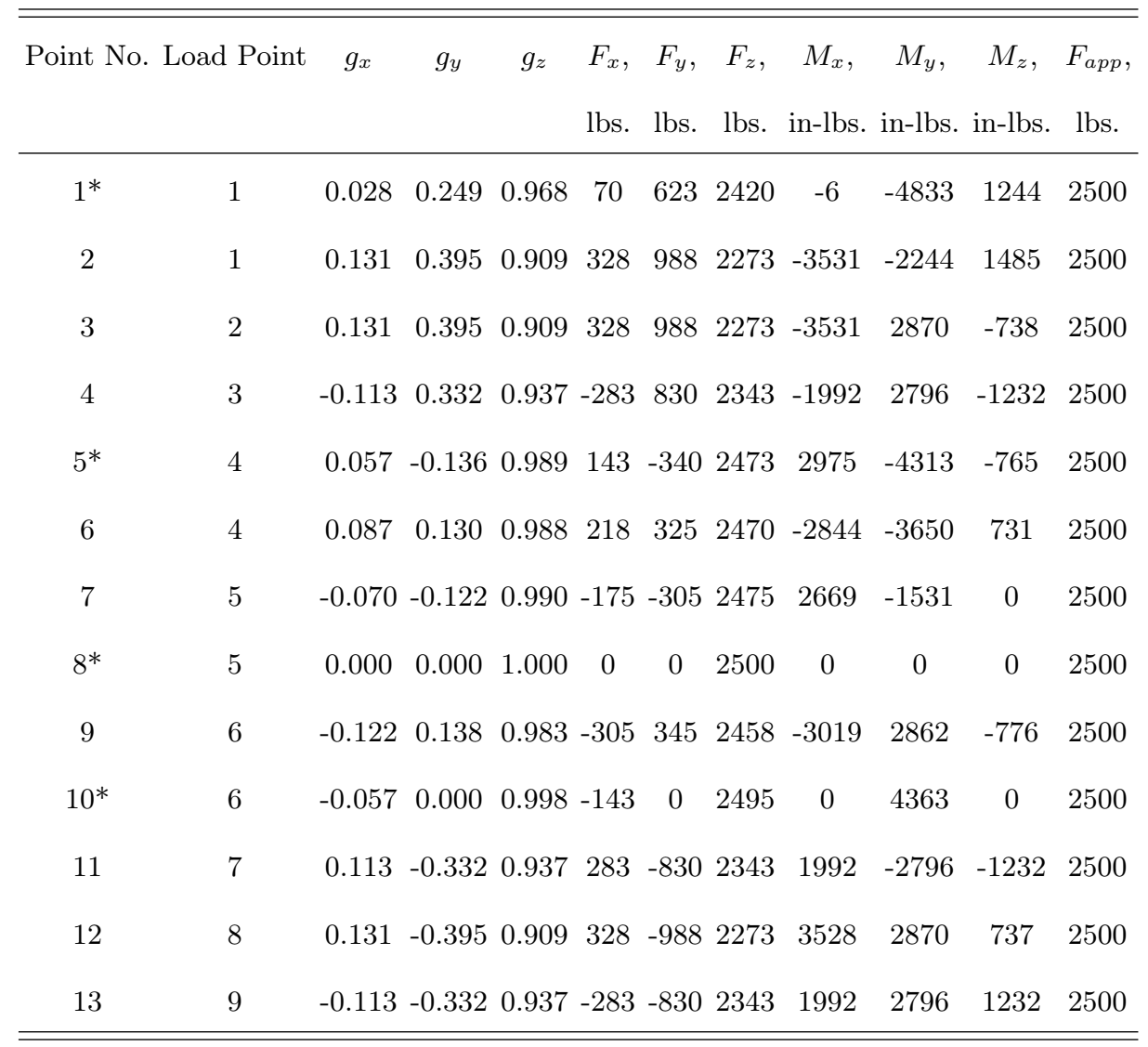

*Note: Asterisk indicates a load combination that is replicated for estimate of experimental error

limits of the balance would have been exceeded at the same orientations given in Table 2. The load schedule included all nine load points and primarily focused on complex, multi-component loads. 
Simple combinations, such as a single-component force, provided a baseline to compare the ILS hardware to readily available hardware, like a knife-edge weight hanger. Replicates of some load combinations were performed to estimate the pure experimental error [2]. Additionally, the load schedule was replicated over several days to assess any day-to-day variability.

\section{Determination of Prediction Intervals}

\section{A. Uncertainty in the NTF-113C Balance Responses}

The first and typically dominant component for estimating balance calibration uncertainties is the residual error from the mathematical model, expressed as MSE. The NTF-113C is calibrated using the NASA LaRC SVS and features a modified Central Composite Design (CCD) for specifying the load schedule. This classic design is perhaps the most popular design for estimating a secondorder response model in many fields. The excellent prediction variance properties and efficient run schedule have been the subject of much discussion in the literature $[2,3]$. The mean square for error is an overall variance estimate resulting from the analysis of variance (ANOVA) and is given in the calibration report for a balance. Values for MSE and residual degrees of freedom $(d f)$ for all components of the the NTF-113C are given in Table 3. Bias error associated with the uncertainty

\section{Table 3 NTF-113C Calibration Model Residual Statistics}

\begin{tabular}{ccccccc}
\hline \hline & $F_{x}$ & $F_{y}$ & $F_{z}$ & $M_{x}$ & $M_{y}$ & $M_{z}$ \\
\hline Residual $d f(n-p)$ & 45 & 49 & 50 & 52 & 55 & 54 \\
$\operatorname{MSE}(\mu V / V)$ & 2.680 & 0.245 & 0.198 & 0.485 & 0.997 & 0.762 \\
\hline \hline
\end{tabular}

in applied loads due to the calibration hardware must be added to MSE for the overall uncertainty. The uncertainty in loads applied by the SVS has been described in Reference 5. Uncertainties in loads applied by the ILS to the NTF-113C balance were computed using this method and are presented in Table 4. 
Table 4 Estimated Bias Errors due to Calibration with SVS

\begin{tabular}{lcccccc}
\hline \hline & $F_{x}$ & $F_{y}$ & $F_{z}$ & $M_{x}$ & $M_{y}$ & $M_{z}$ \\
\hline Avg Variance (lbs.; in-lbs.) & 0.3750 & 0.3803 & 0.3386 & 8.0715 & 7.5861 & 18.0000 \\
Avg Std Dev (lbs.; in-lbs.) & 0.6124 & 0.6167 & 0.5819 & 2.8410 & 2.7543 & 4.2430 \\
Max Std Dev (lbs.; in-lbs.) & 0.6833 & 0.7326 & 0.6692 & 4.3688 & 4.5272 & 4.7060 \\
Min Std Dev (lbs.; in-lbs.) & 0.0817 & 0.0815 & 0.0872 & 0.0832 & 0.0581 & 0.2852 \\
\hline \hline
\end{tabular}

\section{B. Uncertainty in Loads Applied by the ILS}

The weight of the ILS hardware was not subtracted by a tare sequence, but rather included in the calculation of the desired applied load. This requires precise knowledge of the weight and center of gravity of the ILS hardware, which were experimentally determined for both the ILS Mount and remaining ILS subassembly. The mount and the ILS subassembly are moved independently to generate load combinations so that their mass properties were determined separately. The ILS Subassembly consists of the Upper Bearing Mount and the Bearing Cross as seen in Fig. 1. The details of the ILS geometry and measured mass properties may be found in Reference 6 . It was assumed that the force due to the lower bearing mount acts through the load point and therefore the location of the center of gravity is not required. The additional applied force to the balance is the summation of the deadweight load and the weight of the ILS hardware. In addition to precisely determining the mass properties, all critical dimensions that define the moment arms from BMC were evaluated using a calibrated coordinate measuring machine (CMM). The uncertainties associated with all involved measurements are summarized in Table 5.

A Monte-Carlo simulation was developed to combine all the uncertainties, including the tolerance of the CMM-based measurements, uncertainty in the mass of the applied and tare weights, and the uncertainty in the AMS measurements of the gravity vector. The software program incorporated the precise measurements, perturbed with an assumed normal distribution using the standard deviation estimates of Table 5, to calculate the forces at the BMC including the tare weight applied to the balance fixture by the ILS. When using the ILS, uncertainty in the forces is primarily a function of the uncertainty in determining the g-vector. The uncertainty in the applied moments is 
Table 5 Elemental Error Sources for the In-Situ Load System

\begin{tabular}{cccc}
\hline \hline Symbol & Description & Standard Deviation & Units \\
\hline$U_{g_{x}}$ & Projection of Gravity vector on $x$-axis & 0.0001 & $g$ 's \\
$U_{g_{y}}$ & Projection of Gravity vector on $y$-axis & 0.0001 & $g$ 's \\
$U_{g_{z}}$ & Projection of Gravity vector on $z$-axis & 0.0001 & $g$ 's \\
$U_{y_{B M C}, U_{z_{B M C}}}$ & CMM Linear Measurements & 0.0002 & in. \\
$U_{C G x}$ & Center of Gravity Measurement on $x$-axis & 0.0014 & in. \\
$U_{C G y}$ & Center of Gravity Measurement on $y$-axis & 0.0025 & in. \\
$U_{C G z}$ & Center of Gravity Measurement on $z$-axis & 0.0011 & lbs. \\
$U_{F W}$ & Force due to Precision Weights & $0.01 \% \mathrm{~F} . \mathrm{S}$. & lbs. \\
& Force due to ILS Components $<5$ lbs. & 0.00022 & lbs. \\
\hline \hline
\end{tabular}

a function of the moment arm lengths in addition to the forces and as a result, additional variability is introduced. A pseudo-code for the Monte Carlo approach for one design point (load combination) is given below with comments.

Begin

For $i=1$ to 1000 iterations

$\%$ Uncertainty added to Gravity Vector, nrand computes a random value from a normal $\%$ distribution using a standard deviation of $\mathrm{Ug}$ and mean of zero. $\mathrm{G}=[\mathrm{gx}, \mathrm{gy}, \mathrm{gz}]+\mathrm{nr}$ and $(\mathrm{Ug})$

$\%$ Uncertainty added to distances from BMC to ILS Mount (mt) and ILS subassembly (sub) $\mathrm{dBMCmt}=[\mathrm{CGx}, \mathrm{CGy}, \mathrm{CGz}] \mathrm{mt}+[\mathrm{nrand}(\mathrm{UCGx}, \mathrm{UCGy}, \mathrm{UCGz})] \mathrm{mt}+[\mathrm{xBMC}, \mathrm{yBMC}, \mathrm{zBMC}] \mathrm{mt}$

$+[\mathrm{nrand}(\mathrm{Ux}, \mathrm{Uy}, \mathrm{Uz})] \mathrm{mt}$ 


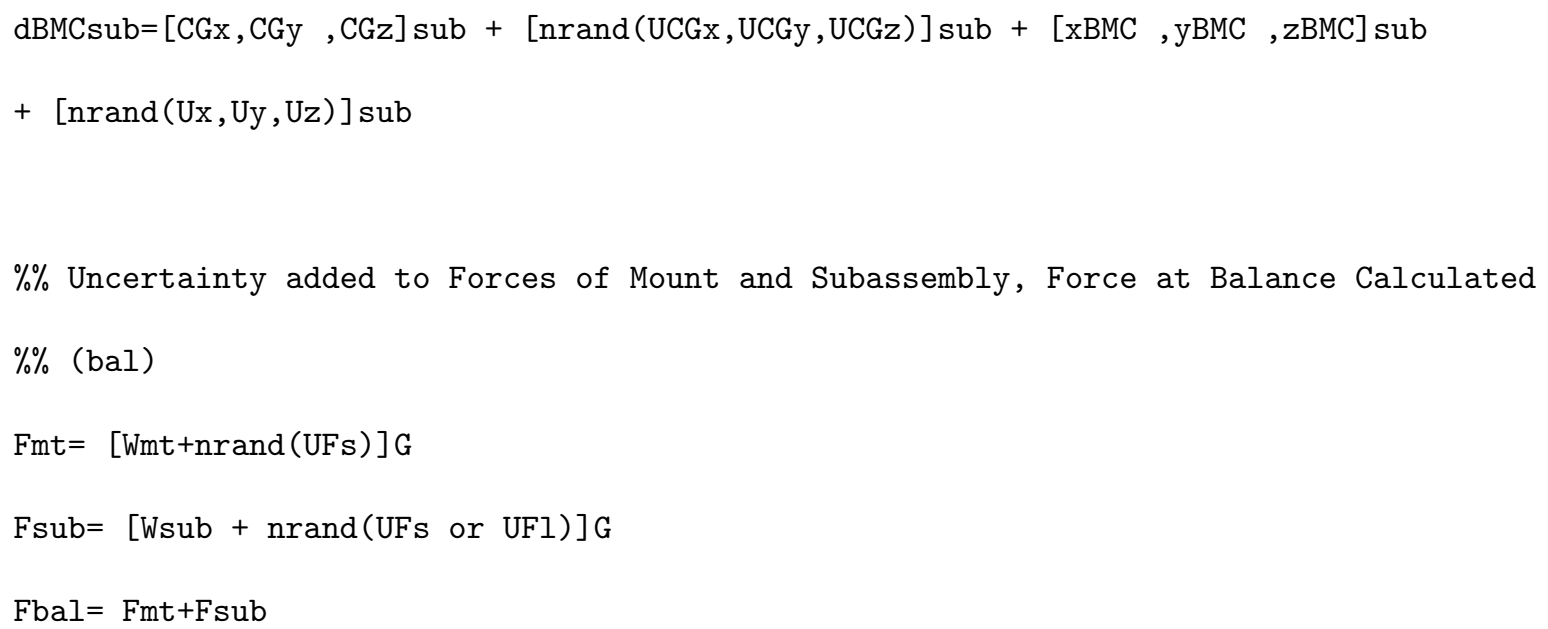

$\%$ Store $\mathrm{Mbal}$ and $\mathrm{Fbal}$ in array

If $i>1000$ go to $*$

If $i<1000$ begin next iteration $i=i+1$

$\% *$ Calculate the mean and standard deviation of each component force and moment using $\%$ the 1000 trials

End

Squaring the overall standard deviation $(\sigma)$ in each response calculated for each of the runs provides an absolute bias estimate for the ILS applied loads, $\sigma_{\text {bias-applied }}^{2}$ Each orientation of the ILS provides a different load combination (design point) and requires a separate Monte Carlo simulation. 
Results from this study are summarized in Table 6 with upper and lower bounds from different load combinations. The most accurate value for $\sigma_{\text {bias-applied }}^{2}$ is found by using the computed value at the given orientation. An engineering compromise may be to look at the bias over all orientations tested and take the average. This was the approach taken in this case study using the orientations of Table 2; average values are given in Table 6 . Conversion from calculated forces and moments

Table 6 Bias in ILS Applied Loads over Study Design Points

\begin{tabular}{lcccccc}
\hline \hline & $F_{x}$ & $F_{y}$ & $F_{z}$ & $M_{x}$ & $M_{y}$ & $M_{z}$ \\
\hline Avg Variance (lbs.; in-lbs.) & 0.0627 & 0.3918 & 0.1184 & 5.5590 & 0.0653 & 5.3490 \\
Avg Std Dev (lbs.; in-lbs.) & 0.2503 & 0.6259 & 0.3441 & 2.3580 & 0.2556 & 2.3130 \\
Max Std Dev (lbs.; in-lbs.) & 0.2676 & 0.8999 & 0.3720 & 2.7400 & 0.2834 & 2.5480 \\
Min Std Dev (lbs.; in-lbs.) & 0.2293 & 0.0006 & 0.3152 & 2.1380 & 0.2341 & 2.1390 \\
\hline
\end{tabular}

in engineering units to response voltages is then done using balance primary sensitivities. Table 6 provides a summary of computed bias values averaged over the complete range of ILS orientations for this study. The average variance (first row of Table 6) was used for $\sigma_{\text {bias-applied }}^{2}$ in subsequent prediction interval calculations.

\section{Prediction Interval Capture Probability for the ILS Case Study}

All terms in the prediction interval of Eq. (4) are now known. The user may choose to convert the response voltages back to units of force and moment for ease of interpretation. Use of balance primary sensitivities for this conversion represents a reasonable engineering compromise.

The load combinations given in Table 2 were executed and replicated over four days on the NTF-113C balance. Responses from the six strain-gage bridges were recorded and the six balance loads were estimated using the iterative balance reduction process [1]. The most recent calibration matrix for the NTF-113C balance was used to estimate the balance loads. Once the six balance loads were estimated, prediction intervals based on Eq. (4) were applied to the estimated loads. The intervals were compared with the physics-bases calculations of the applied load to check whether the applied load fell within the six prediction intervals. The acceptable error rate, $\alpha$, in Eq. (4) was 
set to 0.05 , which yielded a 95-percent confidence level for a single prediction interval. The overall probability that the six prediction intervals simultaneously captured the known physics-based load was at least $1-(6 \times 0.05)=0.70$ or 70 percent. The Bonferroni adjustment to the error rate was made to account for the simultaneous nature of the prediction intervals [9]. For an overall capture probability of 95 percent, the new error rate, $\alpha^{*}$, based on Bonferroni's method was set to $\alpha^{*}=\alpha / 6$ and $\alpha^{*}$ replaced $\alpha$ in Eq. (4). Table 7 summarizes the capture probabilities from the ILS check-load

Table 7 Summary of Capture Probabilities from Check-Load Study

\begin{tabular}{cccccc}
\hline \hline & & \multicolumn{2}{c}{$\mathbf{9 5 \%}$ Prediction Interval } & \multicolumn{2}{c}{ 2 $\sigma$ Calibration } \\
Date & Total Number of & Number of Captured & Percent & Number of Captured & Percent \\
& Combinations & Points & Captured & Points & Captured \\
\hline $07 / 11 / 2013$ & 156 & 150 & 96.2 & 142 & 91.0 \\
$07 / 12 / 2013$ & 240 & 236 & 98.3 & 212 & 88.3 \\
$07 / 15 / 2013$ & 126 & 119 & 94.4 & 113 & 89.7 \\
$07 / 23 / 2013$ & 126 & 121 & 96.0 & 106 & 84.1 \\
\hline Total & 648 & 626 & 96.6 & 573 & 88.4 \\
\hline \hline
\end{tabular}

testing. A total of 108 load combinations $(108 \times 6=648$ points $)$ were performed over the four-day period. The last column of Table 7 compares the capture rate for the two standard deviations of the back-computed residuals to the capture rate from the newly-derived prediction interval method. This comparison is made due to the perceived popularity of using the former interval for checkloading. The overall capture rate using the back-computed $2 \sigma$ method was 88.4 percent versus the prediction interval presented here, which captured 96.6 percent of the check-load points. As mentioned previously, the capture rate was expected to be at least 95 percent.

Table 8 shows a representative single check-load and the comparison between the actual applied load and the estimated load from the six balance bridges. The load combination in the example is point number 12 in Table 2 . It is worth noting that applying the exact combination as stated in Table 2 is not important since an AMS measures the orientation of the balance, and the applied forces and moments are easily calculated from the physics-based equations given by Eqs. (5) and (6). In this example, the actual load for five of the six components falls within the prediction 
Table 8 Example Comparison of Actual and Estimated Loads with Prediction Intervals

\begin{tabular}{ccccc}
\hline \hline Component & Estimated & Actual & $95 \%$ Prediction & $2 \sigma$ Calibration \\
& Load, & Load, & Interval Half-Width, & Interval Half-Width, \\
& lbs. or in-lbs. & lbs. or in-lbs. & lbs. or in-lbs. & lbs. or in-lbs. \\
\hline$F_{x}$ & 324.60 & 325.10 & 2.90 & 1.32 \\
$F_{y}{ }^{2}$ & -986.39 & -982.55 & 3.88 & 2.40 \\
$F_{z}{ }^{2}$ & 2277.47 & 2275.00 & 4.71 & 2.10 \\
$M_{x}$ & 3497.50 & 3497.93 & 28.94 & 20.44 \\
$M_{y}$ & 2817.59 & 2821.24 & 28.63 & 14.18 \\
$M_{z}{ }^{1,2}$ & 721.83 & 742.39 & 19.44 & 9.00 \\
\hline \hline
\end{tabular}

*Note: $\mathrm{A}^{1}$ indicates an applied load outside of the $95 \%$ prediction interval and $\mathrm{a}^{2}$ indicates an applied load outside of the $2 \sigma$ calibration interval

intervals on the estimated load. It is noted that the prediction intervals for the moments are larger due to additional uncertainties in the moment arm lengths. Table 8 also provides a comparison of the prediction interval half-widths of this study and half-width intervals computed using two times the standard deviation of the back-calculated residuals [1]. Using the $2 \sigma$ calibration intervals, only three of the six components were captured.

\section{Conclusion}

The development of the ILS hardware naturally led to the need for a rigorous estimate of the prediction interval for a given combined loading. Accounting for all of the contributing sources of variation for a prediction interval calculation represents a formidable accounting task. The honest assessment of the newly-derived prediction interval provided a capture rate for loadings tested of over 96 percent. The use of bias values derived from averages over a range of the applied load combinations used during calibration and check-loading may prove adequate based on these initial results. Future work may address the potential differences in prediction interval point capture afforded through detailed bias assessments at each individual load combination. In addition, the method should be proven with other balance and check-load hardware combinations. 


\section{Acknowledgments}

The authors would like to express their sincere appreciation to the individuals that have contributed to the many aspects of this project. In particular, the authors would like to recognize the following for the critical contributions: J. Greg Jones for his extensive expertise, knowledge, and dedication to balance calibrations, Ray Rhew for his valuable input on force measurement system design and characterization, Dr. Peter Parker for his insight on the SVS and consultation during the development of the ILS, and Michael Acheson for his initial work with the ILS. This work has been supported and funded by the National Force Measurement Technology Capability (NFMTC) under NASA's Aeronautics Test Program (ATP). 
[1] AIAA, "Recommended Practice: Calibration and Use of Internal Strain Gage Balances with Application to Wind Tunnel Testing," Tech. Rep. R-091-2003, AIAA, 2003.

[2] Montgomery, D., Design and Analysis of Experiments, John Wiley \& Sons, New York, 7th ed., 2009.

[3] Myers, R. H. and Montgomery, D. C., Response Surface Methodology, John Wiley \& Sons, 2nd ed., 2002.

[4] Coleman, H. W. and Steele, W. G., Experimentation, Validation, and Uncertainty Analysis for Engineers, John Wiley \& Sons, New York, 3rd ed., 2009.

[5] Parker, P. and Liu, T., "Uncertainty Analysis of the Single-Vector Force Balance Calibration System," 22nd AIAA Aerodynamic Measurement Technology and Ground Testing Conference, American Institute of Aeronautics and Astronautics, 2002.

[6] Commo, S. A., Lynn, K. C., Toro, K. G., and Landman, D., "Development of the In-Situ Load System for Internal Wind-Tunnel Balances," Journal of Aircraft, 2014.

[7] Parker, P. A., "A Single-Vector Force Calibration Method Featuring the Modern Design of Experiments," 39th AIAA Aerospace Sciences Meeting and Exhibit, No. 2001-170, AIAA, Reno, NV, January 2001.

[8] Parker, P. A. and Finley, T. D., "Advancements in Aircraft Model Force and Attitude Instrumentation by Integrating Statistical Methods," Journal of Aircraft, Vol. 44, 2007, pp. 436-443.

[9] Dean, A. and Voss, D., Design and Analysis of Experiments, Springer, 1999. 